\begin{tabular}{|c|c|}
\hline Title & $\begin{array}{l}\text { Estimation of Gas Permeation Characteristics of UItrahigh Barrier Edge Sealing Materials from A symptotic Solution of } \\
\text { Diffusion Equation }\end{array}$ \\
\hline Author(s) & Shimada, Toshihiro; Y anase, Takashi; Nagahama, Taro; Kanno, Toshiy uki \\
\hline Citation & $\begin{array}{l}\text { Japanese Journal of A pplied Phy sics, 52, 05D A 12-1-05DA 12-3 } \\
\text { https://doi.org/10.7567/JJA P.52.05DA 12 }\end{array}$ \\
\hline Issue Date & $2013-05$ \\
\hline Doc URL & http:/hdl.handle.net/2115/53291 \\
\hline Rights & (c) 2013 The Japan Society of A pplied Physics \\
\hline Type & article (author version) \\
\hline File Information & JJAP_barrier_calc_rev2_after_E.pdf \\
\hline
\end{tabular}

Instructions for use 


\title{
Estimation of Gas Permeation Characteristics \\ of Ultrahigh Barrier Edge Sealing Materials \\ from Asymptotic Solution of Diffusion Equation
}

\author{
Toshihiro Shimada ${ }^{1,2}$, Takashi Yanase ${ }^{3}$, Taro Nagahama ${ }^{1}$, Toshiyuki Kanno ${ }^{4}$ \\ ${ }^{1}$ Division of Materials Chemistry, Faculty of Engineering, Hokkaido University, Sapporo, Hokkaido \\ 060-8628, Japan \\ ${ }^{2}$ JST, CREST, Gobancho, Chiyoda-ku, Tokyo 102-0076, Japan \\ ${ }^{3}$ Frontier Chemistry Center, Faculty of Engineering, Hokkaido University, Sapporo, Hokkaido 060- \\ 8628, Japan \\ ${ }^{4}$ MORESCO, Kobe 650-0047, Japan
}

\begin{abstract}
Materials and structures for water vapor barrier sealing are now actively studied, as the commercialization of organic electronic devices has become a reality. In this paper, we focus on the edge sealing barriers, in which diffusion plays an essential role. In the past, the diffusionlimited gas barrier properties were analyzed in the steady-state approximation, which is never reached within the device lifetime in the application for organic electronics. We analyze them using a simple analytical model. The diffusion before reaching the steady state is a strongly nonlinear process, as is well known, and the length scale of approximately $1-10 \mathrm{~mm}$ is very important when a practical polymer resin is used for the edge seal.
\end{abstract}




\section{Introduction}

Water vapor sealing is very important for organic electronics, since most of the cathode materials and n-type organic semiconductors are vulnerable to water molecules. Efforts to make materials and structures with high gas barrier properties are essential for flexible electronics using organic materials. Gas barrier sealing is currently pursued in two directions. One is the front and substrate seal, or blocking at the surface [Fig.1 (a)]. The other is the edge seal with adhesive functions [Fig. 1(b)]. Since the front seal is technically more difficult and essential in flexible electronics, most of the past research was focused on it. The water vapor transmission rate (WVTR) is a standard value for the characterization of the front barrier coating, and indicates the water mass transmitted through the barrier in a certain time. This definition assumes steady-state permeation of water molecules. The fact that WVTR is an experimentally well-defined value for characterizing the front seal ${ }^{1,2}$ indicates that the permeation through the front seal is a steady-state phenomenon. This is probably because the front barrier coating is thin (usually $<1 \mu \mathrm{m}$ ) but strongly water-blocking because of the deposited inorganic materials, and the pore size of pinholes in the coating is the limiting factor in the permeation. With this assumption, it is understood that the diffusion process thourough the coating is not the rate determining factor, and thus the steady state, which is observable within 1-7 days if a sensitive detector is used, is reached during the measurement ${ }^{2}$.

However, the gas barrier behavior through the edge seal [Fig.1 (b)] might be different from the steady state, because permeation distance is much greater (usually $>1 \mathrm{~mm}$ ) and the sealant contains less-water-blocking polymer resin to allow adhesion. An example of the micrograph of the edge seal material, which is composed of clay mineral flakes embedded in UV-curable epoxy resin, developed by one of the authors (MORESCO) is shown in Fig. 2. The diffusion of gas molecules through the polymer is blocked by the clay mineral flakes and "the meander effect" or "tortured diffusion" is expected.

In this study, we analyze vapor transmission through a thick layer by diffusion, which models the edge seal. The diffusion equation is solved analytically and numerically, and the asymptotic 
behavior is discussed in detail. Gas permeation through polymer composites has been studied for long time ${ }^{3-13}$, but its analysis has been based on the assumption of the steady state. Since the steady state is not reached within the device lifetime during the normal operation of gas barrier edge seals for organic electronics, as discussed in the following, the present results significantly differ from those of previous studies and various implications can be discussed. Thus, we consider this work is worth reporting as a reference for industry sectors, even though the mathematical analysis is very simple and well known.

\section{Mathematical modeling}

First we consider a simple model of one-dimensional diffusion from the outside of the seal to the device side (Fig. 3). The concentration of the permeating gas molecules $(c)$ is a function of time $(t)$ and position $(x)[c(x, t)]$. The outside is in an environment with a certain constant concentration of the permeating gas. Thus, the concentration of the gas molecule at the outer surface $(x=0)$ is constant $\left(c(0, t)=c_{0}\right)$. It should be noted that $c_{0}$ is proportional to the solubility of the gas into the sealing barrier material. The concentration of the gas on the device side is zero at the beginning $[c(d, 0)=0]$. Since the concentration inside the device region should be uniform, $\mathrm{c}(x, t)=\mathrm{c}(d, t)$ for $x$ $>d$. This condition is satisfied if $\partial c(d, t) / \partial t=0$. The gas concentration on the device side at a given time $[c(d, t)]$, which increases as the water vapor diffuses through the barrier, is the value that should be considered. $\quad c(x, t)$ obeys the diffusion equation:

$$
\frac{\partial c}{\partial t}=D \frac{\partial^{2} c}{\partial x^{2}}
$$

where $D$ is the diffusion constant of the gas in the sealing material. With the initial and boundary conditions mentioned above, the solution of the diffusion equation can be approximated

$$
c(x, t) / c_{0}=\operatorname{erfc}\left(\frac{d}{2 \sqrt{D t}}\right)
$$

where "erfc" denotes the error function, 


$$
\operatorname{erfc}(y)=1-\frac{2}{\sqrt{\pi}} \int_{0}^{y} e^{-s^{2}} d s
$$

Equation (2) is valid if $\partial c(d, t) / \partial t$ resulting from it is negligibly small.

The overall behavior of the concentration on the device side, determined by numerical calculation, which is exact within the line width of the curve, is plotted in Fig. 4. We assumed $D=1$ and $d=1$ for simplicity. It should be noted that $c(d, t)$ first increases very slowly $(t<0.2)$, reaches a nearly steady state $(t \sim 2)$, and saturates $(t>5)$. The previous studies dealt with the steady state assuming constant concentrations on each side, which approximately corresponds to $t \sim 2$ in Fig. 4. The implication concerning the gas barrier properties will be discussed in the next section.

\section{Discussion}

Recent requirements for gas barrier sealing are very demanding. In the case of ultrahigh barrier sealing for organic light emitting diodes (OLEDs), the allowed WVTR value is $10^{-6} \mathrm{~g} / \mathrm{m}^{2} /$ day. Considering the experimental finding that the best protecting polymers with $1 \mathrm{~mm}$ thickness show the WVTR value of $10^{0} \sim 10^{-2} \mathrm{~g} / \mathrm{m}^{2} /$ day, it is understood that the required WVTR value is far from that of steady-state permeation. The initial slow permeation region shown in the inset of Fig. 4 should be used for OLED applications. In this region, $t$ is small in Eq.(2), corresponding to a large value of the argument $y$ of $\operatorname{erfc}$ [Eq.(3)].

The asymptotic form of $\operatorname{erfc}$ is given as follows ${ }^{14}$ :

$$
\operatorname{erfc}(y)=\frac{e^{-y^{2}}}{y \sqrt{\pi}} \sum_{n=0}^{\infty}(-1)^{n} \frac{(2 n) !}{n !(2 y)^{2 n}}
$$

which leads to the following:

$$
c(d, t) / c_{0}=\frac{2}{\sqrt{\pi}} \frac{d}{\sqrt{D t}} \exp \left(-\frac{d^{2}}{4 D t}\right)\left(1-\frac{2 D t}{d}+\ldots\right) \approx \frac{2}{\sqrt{\pi}} \frac{d}{\sqrt{D t}} \exp \left(-\frac{d^{2}}{4 D t}\right),
$$

The approximation by Eq. (5) is valid when $c(d, t) / c_{0}<<1$.

Using the reported diffusion constant value of water molecules on epoxy resin $\left(D=10^{-8} \mathrm{~cm}^{2} / \mathrm{s}\right)^{15}$, $c(d, t) / c_{0}$ for small $t$ was calculated from eq. (4) and plotted in Figs. 5(a)- 5(c). If the length of the 
sealing region $(d)$ is $2 \mathrm{~cm}$, water vapor will not be detected for ten days [Fig. 5(a): $c / c_{0}=10^{-100}$ ]. If $d$ $=0.2 \mathrm{~cm}$, water vapor will diffuse into the device region considerably $\left(c / c_{0}=10^{-2}\right)$ within two or three days. If $d=0.02 \mathrm{~cm}$, the diffusion is almost complete $\left(c / c_{0} \sim 1\right)$ in $1 \mathrm{~h} . \quad$ In Figs. 5(b) and 5(c), where the numerical results of erfc were available, they were plotted to show the error in approximation of Eq.(5). These estimations of barrier properties are in reasonably agreement with our finding that edge sealing for OLED requires a substantial length of much more than $1 \mathrm{~mm}$. This analysis indicates that it is very important to measure WVTR as the function of time and length (and thickness) of the sealant, and to derive the diffusion constant $D$ from the fitting to enable a rationalized design of the edge seal dimensions.

Next we discuss the implication of the present analysis on the composite material shown in Fig. 2. The composite barrier resin gives an OLED device lifetime more than 2 times longer than other commercial edge sealants prepared using similar polymers but different inorganic fillers, which indicates that the effect of inorganic filler is important to the barrier property. Suppose that inorganic flakes having the aspect ratio $\alpha$ (height / width, $<1$ ) are mixed in the polymer to a volume fraction of $f$. Then the diffusion must proceed while avoiding the flakes; this is called the "meander effect" or "tortured diffusion effect". The effective diffusion length $d$ ' is given by the geometrical consideration $^{8,16}$

$$
d^{\prime} \simeq \alpha^{-1} f d,
$$

where $d$ is the actual length of the edge seal. Assuming $\alpha$ is 0.01 and $f$ is $50 \%, d^{\prime}$ becomes $50 d$. The enhancement factor for the diffusion-limited barrier is more than single power of $d$, as indicated in Fig. 5, and the resulting improvement of the sealing properties can be very large.

The gas permeation rate in the inorganic-organic composite has been analyzed assuming the steady state ${ }^{3-4}$. The permeation rate was proportional to the diffusion constant in that case, which is different from the present analysis. The apparent diffusion constant in the composite is modified to the value $D^{\prime}$, which is given by a geometrical consideration of the meander effect as follows ${ }^{3-4}$ :

$$
D^{\prime}=D \alpha^{2}(1-f) f^{-2}
$$

where $D$ is the diffusion constant of the gas molecule in the polymer, and $\alpha$ and $f$ have the same 
meanings as above. Assuming $\alpha=0.01$ and $f=50 \%$ will reduce the permeation by 5000 . Although this value is large enough to account for the experimental results ${ }^{3-5}$, the effect of inorganic flakes can be much greater in the sub-steady-state diffusion discussed above. Further drastic reduction in the length of the edge seal may be achieved, if the materials and structures are properly designed.

\section{Conclusions}

We have presented a mathematical analysis of the gas barrier when diffusion is the limiting factor, which is important for the edge seal of organic devices. In the region of ultrahigh barriers used for OLED applications, diffusion never reaches the steady state and it should be analyzed carefully. We found that the concentration of the permeated gas on the device side is strongly dependent on the diffusion distance. This behavior reveals the dominant contribution to be the effect of inorganic flake filler blended in the polymer adhesive, which should be considered in the design of the edge seal.

\section{Acknowledgments}

The present work was partly supported by MEXT KAKENHI Grant Number 23656001 and a Supporting Industry Grant from METI, Japan. 
References

1. P.A.Carcia, R.S.McLean, M.H.Reilly, Appl.Phys.Lett. 89 (2006) 031915.

2. T. Shimada, Y. Takahashi, T. Kanno, Appl. Phys. Exp. 3 (2010) 021701.

3. E.L. Cussler, S.E. Hughes, W.J. Ward, R. Aris, J. Memb. Sci. 38 (1988) 161.

4. D.M. Eitzman, R.R. Melkote, E.L. Cussler, AIChE J. 42 (1996) 2.

5. K.S. Triantafyllidis, P.C. LeBaron, I. Park, T.J. Pinnavaia, Chem. Mater. 18 (2006) 4393.

6. Y. Zhang, Q. Liu, Q. Zhang, Y. Lu, Appl. Clay Sci. 50 (2010), 255.

7. N. Lepot, M.K. Van Bael, H. Van den Rul, J. D'Haen, R. Peeters, D.Franco, J. Mullens, J. Appl. Polymer Sci. 120 (2011) 1616.

8. W-S. Jang, I. Rawson, J.C. Grunlan, Thin Solid Films 516 (2008) 4819.

9. J.P.G. Villaluenga, M. Khayet, M.A. Lopez-Manchado, J.L. Valentin, B. Seoane, J.I. Mengual, Eur. Polymer J. 43 (2007) 1132.

10. J. M. Herrera-Alonso, E. Marand, J.C. Little, S.S. Cox, J. Memb. Sci. 337 (2009) 218.

11. R.D.Maksimov, A. Lagzdins, N. Lilichenko, E. Plume, Polymer Eng. Sci 49 (2009) 2421

12. K.K. Mokwena, J. Tang, M.-P. Laborie, J. Food Eng. 105 (2011) 436.

13. M. Frounchi, S. Dadbin, Z. Salehpour, M. Noferesti, J. Memb.Sci. 282 (2006) 142.

14. Mathematics of Diffusion, 2nd ed. by J. Frank, Oxford Univ. Press, London, 1980.

15. K.Wagner, G.Grundmeier, Int. J. Adhesion \& Adhesives 24 (2004) 193.

16. L.E. Nielsen, J.Macromol. Sci. Chem. A1 (1967) 929. 
Figure captions

Fig. 1: Front seal and edge seal of organic electronic devices.

Fig.2: SEM image of epoxy resin containing high concentration of layered clay flakes in gas barrier edge seal.

Fig.3: Model of gas barrier edge seal. A: Outside atmosphere. B: Edge seal (thickness d). C: Device region.

Fig. 4: Overall behavior of solution $c(d, t)$ of diffusion equation when $D=1$ and $d=1$. Inset is shows enlargement of the small $t$ region.

Fig. 5: Estimation of gas permeation in the initial stage of diffusion-limited edge seal. Solid lines: numerical solutions. Dotted lines: approximation by Eq. (5). It was assumed that $D=10^{-8} \mathrm{~cm}^{2} / \mathrm{s}$. 


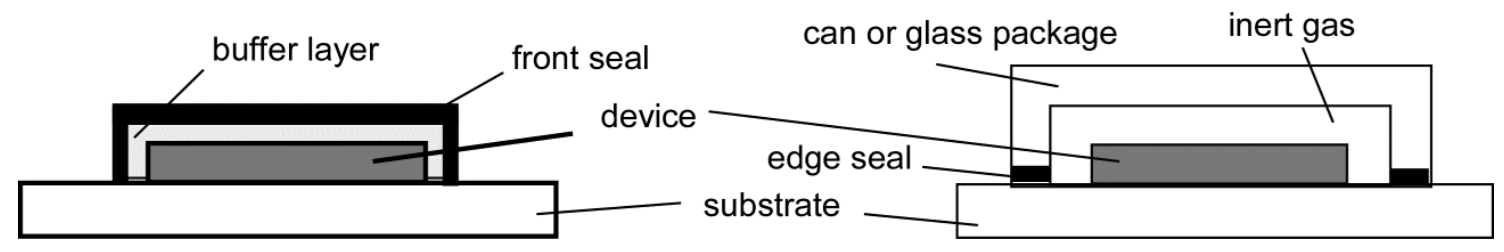

Fig. 1: Front seal and edge seal of organic electronic devices. 


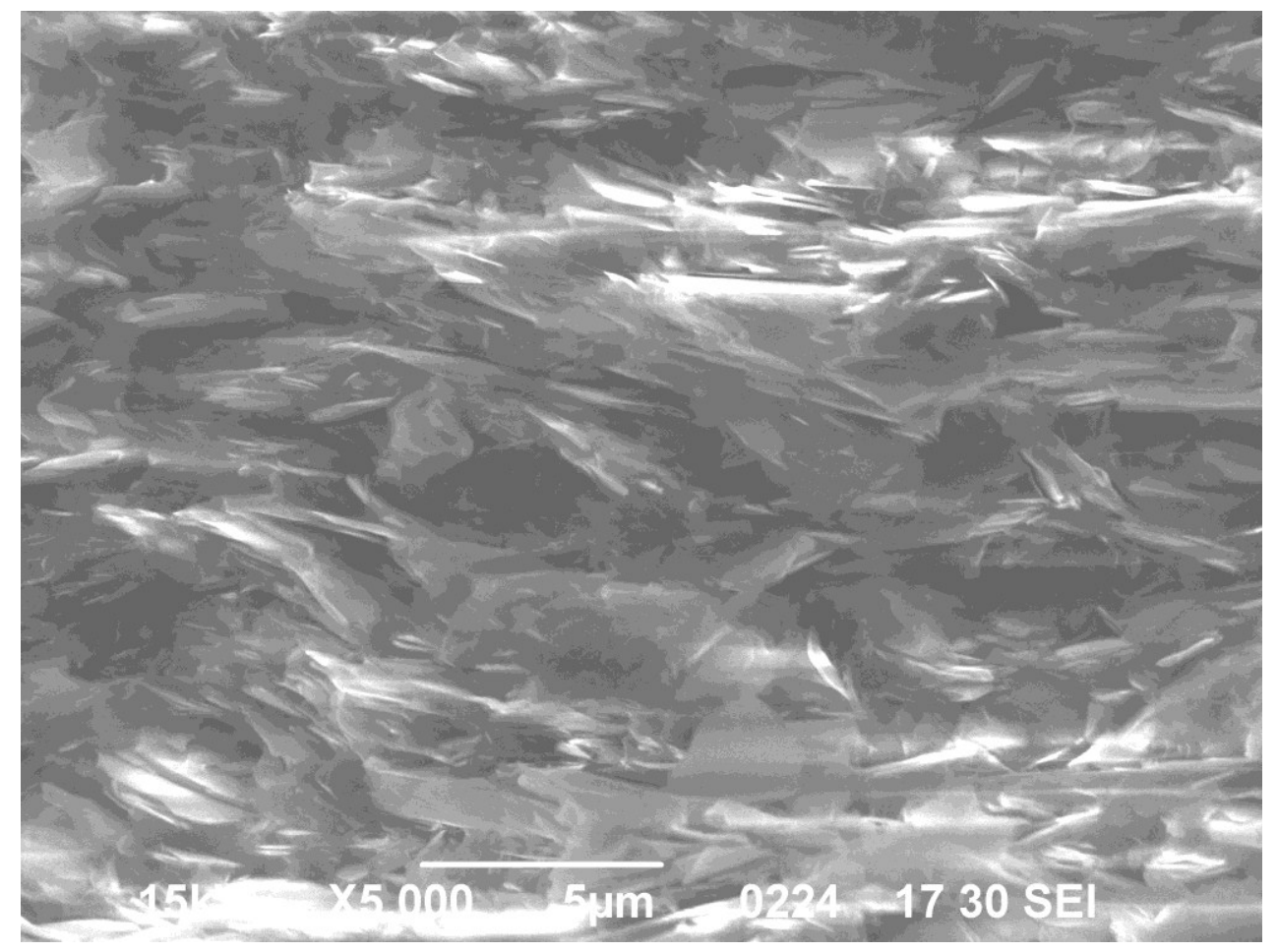

Fig.2: SEM image of epoxy resin containing high concentration of layered clay flakes in gas barrier edge seal. 


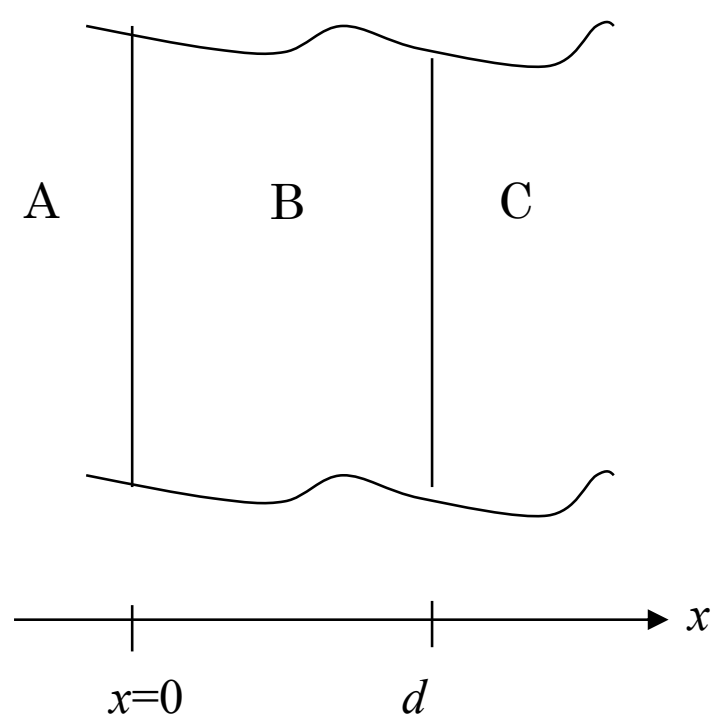

Fig.3: Model of gas barrier edge seal. A: Outside atmosphere. B: Edge seal (thickness d). C: Device region. 


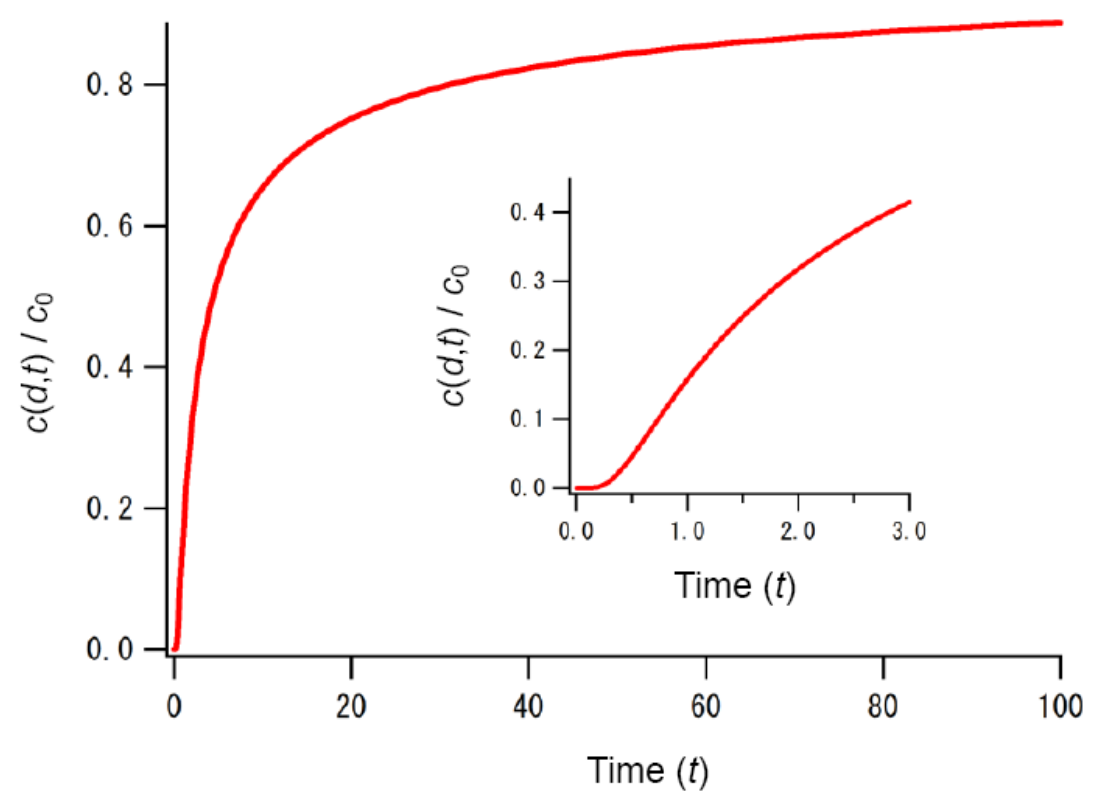

Fig. 4: Overall behavior of solution $c(d, t)$ of diffusion equation when $D=1$ and $d=1$. Inset is shows enlargement of the small $t$ region. 

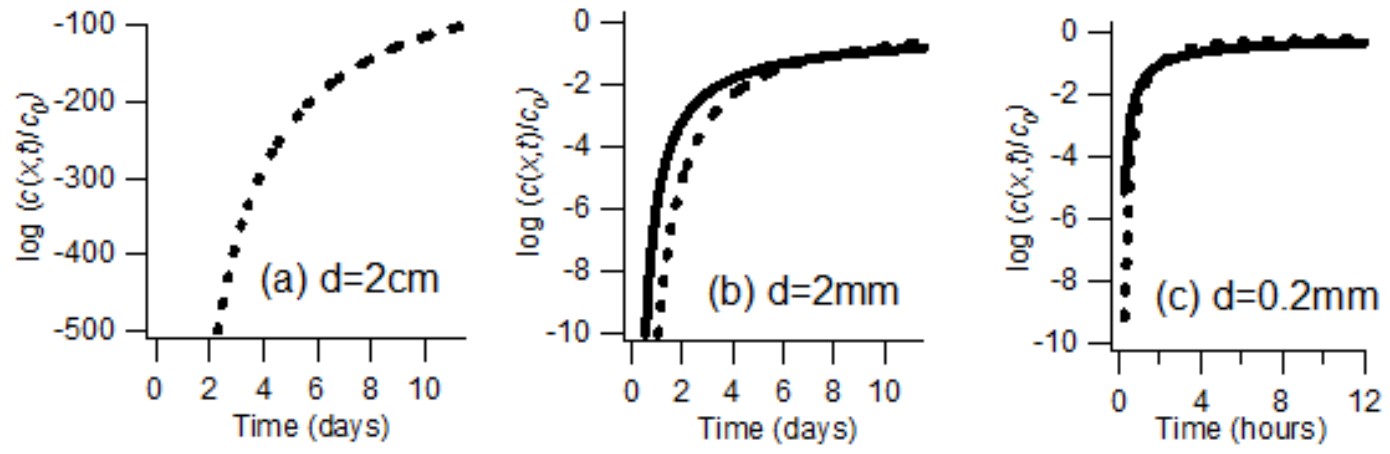

Fig. 5: Estimation of gas permeation in the initial stage of diffusion-limited edge seal. Solid lines: numerical solutions. Dotted lines: approximation by Eq. (5). It was assumed that $D=10^{-8} \mathrm{~cm}^{2} / \mathrm{s}$. 